\title{
Electron Energy-Loss Spectroscopy of Alternative Gate Dielectric Stacks
}

\author{
S. Stemmer*, D. Klenov*, Z. Chen*, J.-P. Maria**, A. I. Kingon**, D. Niu***, G. N. \\ Parsons $* * *$ \\ * Department of Mechanical Engineering and Materials Science, Rice University, \\ Houston, TX 77005-1892 \\ ** Department of Materials Science and Engineering, North Carolina State University, \\ Raleigh, NC 27695-7919 \\ *** Department of Chemical Engineering, North Carolina State University, Raleigh, NC \\ 27695-7905
}

Continued scaling of silicon technology ( Moore s Law ) requires a paradigm shift in the materials used as gate dielectric in complementary metal-oxide-semiconductor (CMOS) devices. In the near future, the $\mathrm{SiO}_{2}$ thickness is projected to be thinner than $1 \mathrm{~nm}$. In this thickness range tunneling currents through the $\mathrm{SiO}_{2}$ become unacceptably high. Currently, alternative dielectrics, such as $\mathrm{ZrO}_{2}, \mathrm{HfO}_{2}, \mathrm{Y}_{2} \mathrm{O}_{3}$ and their alloys with $\mathrm{SiO}_{2}$ or $\mathrm{Al}_{2} \mathrm{O}_{3}$, a re being investigated to replace $\mathrm{SiO}_{2}$. These oxides have greater dielectric constants $(k)$ than $\mathrm{SiO}_{2}$, and are potentially stable in contact with silicon. High-resolution analytical capabilities afforded by scanning transmission electron microscopy techniques are essential in analyzing the interface and bulk stability of these ultrathin $(<5 \mathrm{~nm})$ layers.

Here, we apply high-resolution electron energy-loss spectroscopy in combination with atomic resolution $\mathrm{Z}$-contrast imaging to investigate the stability of alternative gate dielectric layers at high temperatures, and under reducing and oxidizing conditions. Zcontrast images are used to image the chemical homogeneity of the layers and to position the probe for electron energy-loss spectroscopy (EELS). EELS is used to measure composition and bonding across the gate dielectric with sub-nanometer spatial resolution. We use oxygen K-edges and $\mathrm{Si}$ L-edges to investigate interfacial $\mathrm{SiO}_{2}$ and silicate formation. In addition, the near-edge fine-structure of these edges and comparison with bulk reference spectra are used to fi ngerprint phase formation and nonstoichiometry. Conventional high-resolution transmission electron microscopy is used to investigate crystallization.

Using the combination of these methods we are able to determine the stability of these ultrathin layers with a-priori unknown structures. For example, we show that $\mathrm{ZrO}_{2} / \mathrm{Si}$ layers annealed under moderately oxidizing conditions (oxygen partial pressure $>\sim 10^{-4}$ torr), form a low- $k$ interfacial $\mathrm{SiO}_{2}$ layer through oxygen diffusion through the $\mathrm{ZrO}_{2}$ and silicon consumption at the interface. Layers annealed under moderately reducing conditions (oxygen partial pressure $\sim 10^{-5}$ torr) do not show extensive $\mathrm{SiO}_{2}$, formation, whereas layers annealed under even lower oxygen partial pressures $\left(\sim 10^{-7}\right.$ torr $)$ form an interfacial silicide, consistent with predictions from thermodynamic estimates. In contrast to $\mathrm{ZrO}_{2}$ layers, $\mathrm{CVD}$ grown $\mathrm{Y}_{2} \mathrm{O}_{3}$ films show extensive silicate formation upon annealing, through Si diffusion into the dielectric. We show that thin films transform to 
an amorphous yttrium silicate upon annealing, whereas thicker films form an interfacial silicate and crystalline $\mathrm{Y}_{2} \mathrm{O}_{3}$ on the surface. We will discuss possible mechanisms, in particular the role of crystallization and Si diffusion to explain the observed results. We also show that pre-nitridation of the Si surface impedes the Si diffusion.

$1(\mathrm{a})$

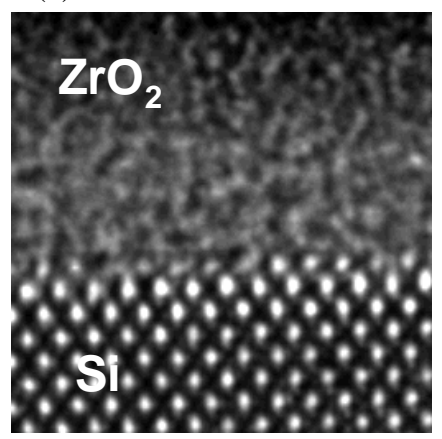

$1(\mathrm{~b})$

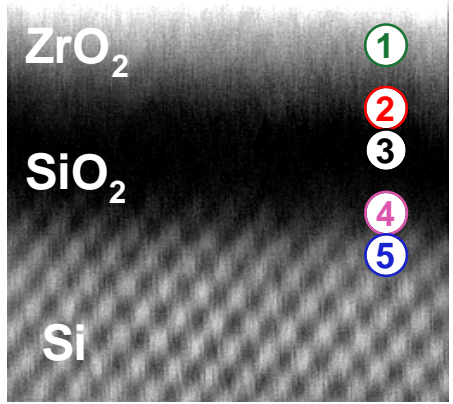

$1(\mathrm{c})$

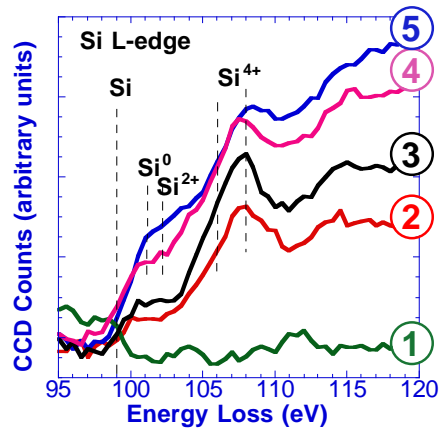

Figure 1: (a) Conventional HRTEM image of an as-deposited $\mathrm{ZrO}_{2}$ film, (b) Z-contrast image and (c) EELS spectra through the thickness of the gate stack. No Si can be detected in the $\mathrm{ZrO}_{2}$ layer. Note the atomic number sensitivity of the Z-contrast image that clearly shows the interfacial $\mathrm{SiO}_{2}$.

2(a)

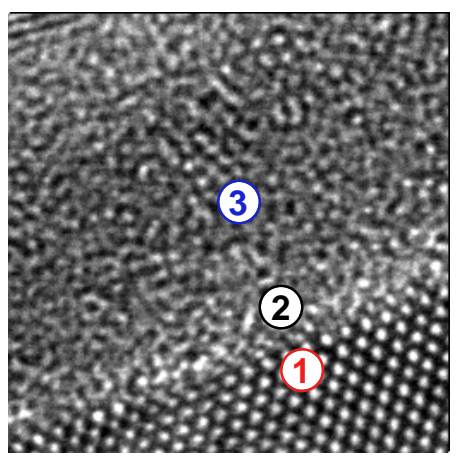

3(a)

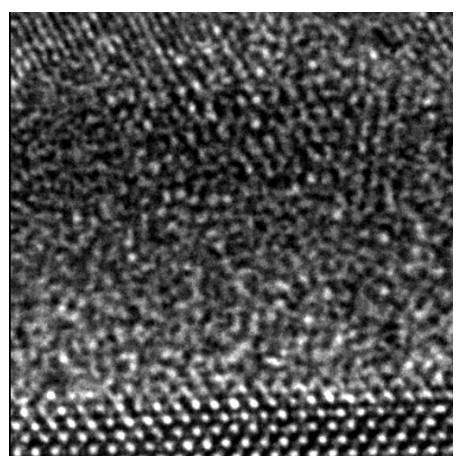

2(b)
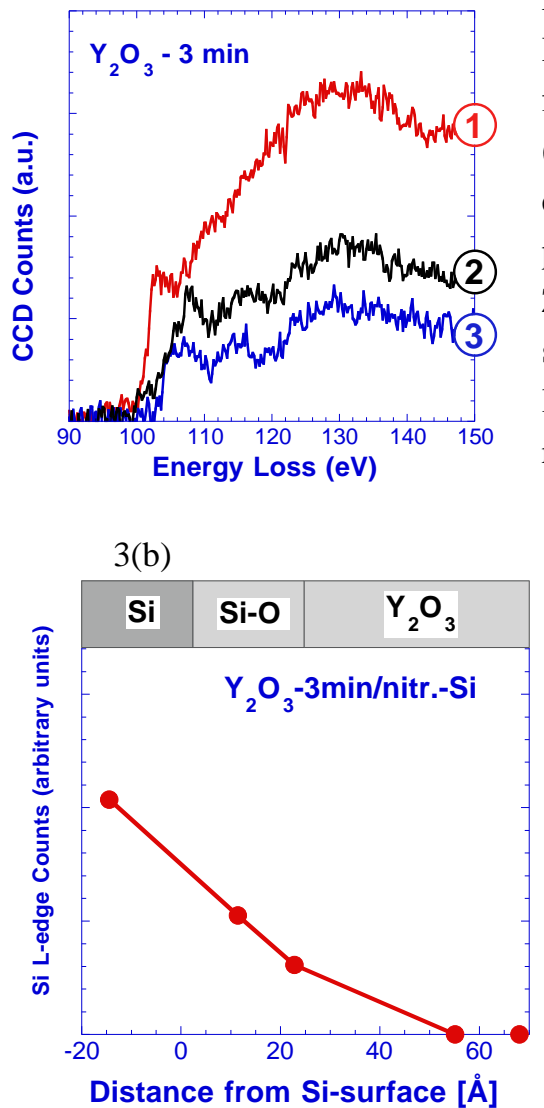

Figure 2: (a) Conventional HRTEM image of an $\mathrm{Y}_{2} \mathrm{O}_{3}$ film deposited on bare silicon. (b) Fine structure of Si Ledges recorded at different positions in the corresponding Z-contrast image (not shown) shows an interfacial $\mathrm{SiO}_{2}$ layer and that the film has reacted to a $\mathrm{Y}$-silicate.

Figure 3: (a) Conventional HRTEM image of an $\mathrm{Y}_{2} \mathrm{O}_{3}$ film deposited on nitrogen plasma pretreated silicon shows that the upper part of the film crystallizes.

(b) Si profile shows that the crystallized film contains no Si.

This research was supported by the SRC/Sematech Front End Process Center. 